\title{
Hipertensão Pulmonar em Lactente Associada a Pulmão em Ferradura: Relato de Caso
}

\author{
Pulmonary Hypertension in Infants Linked to Horseshoe Lung: Case Report
}

\author{
Juliana Rodrigues Neves,2,3, Santiago Raul Arrieta², Catarina V. Cavalcanti ${ }^{1}$, Sandra S. Mattos ${ }^{3}$ \\ Pronto-Socorro Cardiológico de Pernambuco - PROCAPE'; Instituto de Medicina Integral Prof. Fernando Figueira - IMIP2; Unidade de \\ Cardiologia Materno-Fetal - UCMF ${ }^{3}$, Recife, PE - Brasil
}

Relatamos caso de lactente jovem com desconforto respiratório precoce e hipertensão pulmonar, diagnosticado como variante de pulmão em ferradura, e revisamos literatura a cerca desta rara malformação pulmonar e suas repercussões cardíacas e hemodinâmicas.
This is the case report of a young infant with early respiratory distress and pulmonary hypertension, diagnosed as a variant of horseshoe lung and we have reviewed the literature to seek information about this rare pulmonary malformation and its cardiac and hemodynamic repercussions.

\section{Introdução}

Pulmão em ferradura é uma anomalia congênita rara definida como fusão dos segmentos póstero-inferiores dos pulmões. O acometimento do pulmão direito com hipoplasia é mais frequente e, na maioria dos casos, é associado à síndrome da cimitarra. $\mathrm{O}$ acometimento do pulmão esquerdo é excepcional e relacionado à pior evolução, assim como presença de malformações cardíacas associadas e hipertensão pulmonar ${ }^{1,2}$.

Relatamos caso de lactente jovem encaminhado a centro de cardiologia pediátrica por episódios de cianose.

\section{Relato de caso}

Trata-se de lactente de três meses de vida, sexo feminino, encaminhada a centro de referência de cardiologia pediátrica por apresentar dispneia desde o nascimento e episódios frequentes de cianose. Após nascimento, a paciente apresentou hipóxia leve e desconforto respiratório nas primeiras horas de vida, necessitando suporte com ventilação mecânica assistida. Permaneceu em unidade de terapia intensiva (UTI) neonatal por 30 dias e obteve alta hospitalar aos 40 dias de vida, ainda com desconforto respiratório e dificuldade alimentar. Aos dois meses de vida, apresentou episódio de cianose, tendo sido diagnosticado pneumonia à esquerda, necessitando suporte ventilatório por 04 dias e antibioticoterapia específica. Como

\section{Palavras-chave}

Cardiopatias congênitas, hipertensão pulmonar, lactente.

Correspondência: Juliana Rodrigues Neves •

Av. Bernardo Vieira de Melo, 4989/1401 - Candeias - 54440-620 -

Jaboatão dos Guararapes, PE - Brasil

E-mail: ju_neves@cardiol.br, ju.neves2000@gmail.com

Artigo recebido em 20/02/08; revisado recebido em 02/08/09;

aceito em 30/11/09. não houve melhora do quadro após terapia e persistindo com episódios frequentes de cianose, foi encaminhada a serviço terciário em cardiologia pediátrica.

À admissão, apresentava-se com estado geral comprometido, taquidispneica em grau moderado, com leve cianose central sob cateter nasal de oxigênio $\left(\mathrm{O}_{2}\right) 3 \mathrm{l} / \mathrm{min}$. À inspeção do tórax, evidenciava-se abaulamento de precórdio e hiperdinamismo, além de intenso desconforto respiratório. À ausculta cardíaca, encontrava-se em ritmo cardíaco regular, taquicárdica, com hiperfonese do componente pulmonar de segunda bulha, sem sopros. À ausculta pulmonar, evidenciavase diminuição do murmúrio vesicular à esquerda e estertores subcrepitantes difusos, além de discreto estridor inspiratório. Os pulsos eram presentes e simétricos. No abdome, palpava-se o fígado a 4,0 cm do rebordo costal direito.

A paciente foi admitida em UTI cardiopediátrica para estabilização hemodinâmica, onde foi iniciada ventilação não invasiva com fração inspirada de $\mathrm{O}_{2}$ de 0,5 , furosemida de $3,0 \mathrm{mg} / \mathrm{kg} /$ dia e dobutamina de $5,0 \mu \mathrm{g} / \mathrm{kg} / \mathrm{min}$. Evoluiu com estabilização clínica, desmame de suporte ventilatório e inotrópico, de tal modo que, no 5ำ dia após admissão, encontrava-se em ar ambiente com taquidispneia leve, mantendo saturação de $\mathrm{O}_{2}$ em torno de $96 \%$, em uso de furosemida de 3,0 mg/kg/dia e digoxina de $0,01 \mathrm{mg} / \mathrm{kg} / \mathrm{dia}$. Foi então iniciada investigação clínica.

A radiografia do tórax mostrava redução do volume do pulmão esquerdo, com desvio das estruturas do mediastino para este lado (Figura 1a). Trazia do hospital de origem tomografia computadorizada de tórax, cuja avaliação local diagnosticava hipoplasia de pulmão esquerdo. Análise posterior, apesar da má qualidade técnica do exame, detectava também imagem sugestiva de continuidade entre os lobos pulmonares inferiores (Figura 1b).

Ecocardiograma transtorácico identificou aumento de câmaras cardíacas direitas com sinais de hipertensão arterial pulmonar e pequena comunicação interatrial $(\mathrm{ClA})$ do tipo ostium secundum.

Foi realizado cateterismo cardíaco que evidenciou hipertensão arterial pulmonar importante (Tabela 1). 


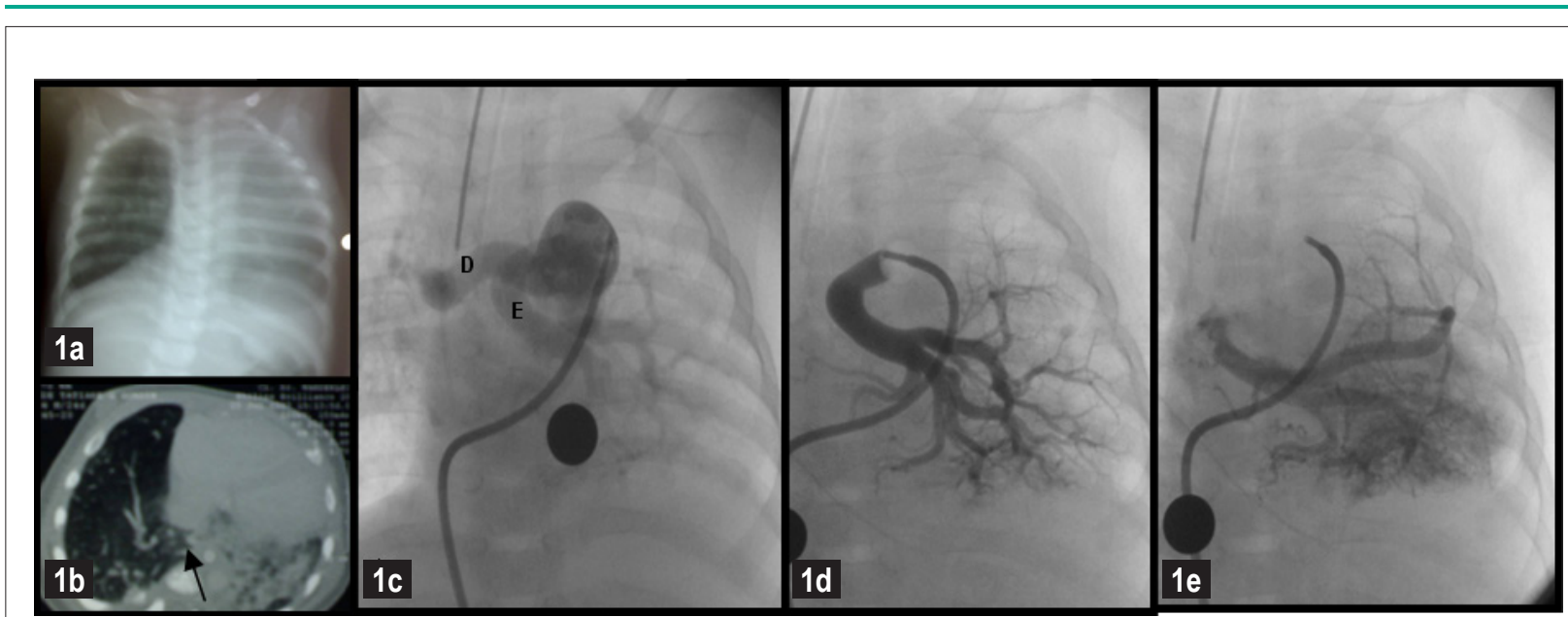

Fig. 1 -1a: Radiografia de tórax em projeção póstero-anterior (PA) - evidencia desvio de estruturas do mediastino para o hemitórax esquerdo; 1b: Tomografia de tórax parênquima pulmonar em linha média do tórax (istmo-seta) e hipoplasia de pulmão esquerdo; 1c: Angiografia de tronco pulmonar em PA mostrando angiografia de tronco pulmonar em PA mostrando a artéria pulmonar direita (D) e o ramo da mesma que se dirige ao istmo para o pulmão esquerdo (E); 1 d: Angiografia seletiva neste ramo para o pulmão esquerdo - curso aberrante e ramificações para linha média; 1e: Retorno venoso do istmo pulmonar, que é paralelo ao ramo arterial, que drena em átrio esquerdo.

Tabela 1 - Pressões e saturação de oxigênio nas câmaras especificadas e cálculos hemodinâmicos

\begin{tabular}{|c|c|c|c|c|}
\hline Cavidade & Pressão sistólica (mmHg) & Pressão diastólica (mmHg) & Pressão média (mmHg) & $\mathrm{SO}_{2}(\%)$ \\
\hline VCS & & & 11 & 60 \\
\hline TP & 94 & 54 & 73 & 65 \\
\hline $\mathrm{AE}$ & & & 12 & 99 \\
\hline \multirow[t]{3}{*}{$\mathrm{AO}$} & 80 & 43 & 56 & 97 \\
\hline & Qp:Qs & $\operatorname{RVP}\left(U W x m^{2}\right)$ & RVS $\left(U W x m^{2}\right)$ & RVP/RVS \\
\hline & 1,09 & 18,80 & 15,10 & 1,246 \\
\hline
\end{tabular}

$\mathrm{SO}_{2}$ - saturação de oxigênio; VCS - veia cava superior; TP - tronco pulmonar; AE - átrio esquerdo; $A O$ - aorta; Qp:Qs - relação de fluxos sistêmico e pulmonar; RVP resistência vascular pulmonar; RVS - resistência vascular sistêmica; UWXm² - unidades Wood por metro quadrado de superfície corporal.

À angiografia, observava-se ramo da artéria pulmonar direita dirigindo-se ao istmo do pulmão esquerdo, não ficando bem evidenciada a origem da artéria pulmonar esquerda (pulmão esquerdo hipoplásico), com o retorno venoso se fazendo paralelamente a este ramo arterial para o istmo pulmonar, drenando em átrio esquerdo (Figuras 1c, 1d e 1e).

Após o cateterismo cardíaco, a criança foi readmitida em UTI para recuperação anestésica e foi iniciada terapia medicamentosa para hipertensão pulmonar com sildenafila nas doses preconizadas. No terceiro dia após o cateterismo, encontrava-se em ar ambiente, com as medicações prescritas anteriormente e aguardando alta para enfermaria, quando apresentou episódio súbito de cianose com bradicardia sinusal que evoluiu para assistolia, sem resposta às manobras de reanimação, evoluindo para o óbito. O episódio foi interpretado pelo plantonista-assistente como "crise de hipertensão pulmonar".

Apesar de ciente da gravidade do quadro e esclarecida quanto à importância da elucidação diagnóstica, a genitora não autorizou estudo anatomopatológico, o que impossibilitou a confirmação anatômica dos dados aqui relatados.

\section{Revisão da literatura e considerações}

O pulmão em ferradura caracteriza-se pela presença de istmo de parênquima pulmonar que se estende através da linha média, conectando as regiões póstero-basais de ambos os pulmões, por trás do coração e anterior à aorta descendente. Desde sua descrição na década de 60 (Spencer, 1962), foram relatados cerca de 40 casos na literatura mundial, em sua maioria associados à hipoplasia do pulmão direito e com frequência associados à síndrome de cimitarra. Há apenas um caso descrito em um feto de 19 semanas, onde o istmo pulmonar estava localizado na porção apical dos pulmões, o que levou a reflexões sobre a origem embriológica desta malformação ${ }^{1-3}$.

Na maioria dos casos, a sintomatologia clínica inicia-se precocemente com problemas pulmonares em crianças jovens, lactentes e neonatos. Desconforto respiratório frequente, pneumonias de repetição e episódios de cianose são os achados mais comuns. Hipertensão pulmonar tem sido implicada na evolução precoce de tais pacientes, apesar de não ser regra ${ }^{1,4}$. A síndrome de cimitarra associa-se em 15\% dos casos com o pulmão em ferradura e, apesar das semelhanças 


\section{Relato de Caso}

morfológicas entre a síndrome com ou sem esta associação, sua apresentação clínica é diferente ${ }^{5}$. Os pacientes com pulmão em ferradura apresentam mais cedo sintomatologia evidente e necessidade precoce de cirurgia, conforme discussão a seguir. Cardiopatias congênitas ocorrem em 25\% dos casos, sendo a comunicação interatrial a mais comum (50\%). Outras lesões incluem comunicação interventricular, persistência do canal arterial, tetralogia de Fallot, drenagem anômala total de veias pulmonares (em caso descrito recentemente em literatura nacional) e coarctação da aorta ${ }^{6,7}$.

Nosso paciente apresentou sintomatologia em fase muito precoce da vida, com hipertensão pulmonar severa, associada à comunicação interatrial e anomalia de drenagem venosa pulmonar, fatores relacionados ao pior prognóstico.

Na radiografia de tórax, observa-se hipoplasia de um dos pulmões e desvio das estruturas do mediastino para o mesmo lado, porém não há achado característico da malformação. Tomografia computadorizada de tórax demonstra hipoplasia pulmonar unilateral com istmo que se estende além da linha média. Geralmente, o pulmão direito é afetado, entretanto, assim como o caso aqui relatado, foram descritos casos de acometimento de pulmão esquerdo, sendo observado ainda que o istmo origina-se do pulmão hipoplásico em todos os $\operatorname{casos}^{2,8}$. A definição do diagnóstico é feita por broncografia ou angiografia quando é definido o suprimento brônquico e vascular anômalo para o istmo pulmonar. O cateterismo cardíaco, além de demonstrar a anatomia vascular arterial, é importante ferramenta na avaliação da drenagem venosa do pulmão afetado, podendo haver ou não alteração e obstrução em seu trajeto, e no diagnóstico e estudo hemodinâmico dos casos associados à hipertensão pulmonar ${ }^{1,2}$.

No caso em questão, a possibilidade diagnóstica foi aventada apenas após o cateterismo cardíaco, quando o tratamento com sildenafila foi instituído na tentativa de minimizar a sintomatologia relacionada à hipertensão arterial pulmonar, a qual parece ter contribuído sobremaneira para o desfecho do caso.

Quando possível, o tratamento cirúrgico está indicado na presença de infecções recorrentes, "curto-circuito" esquerdo-direito significativo (Qp:Qs > 2:1) na presença de defeitos cardíacos associados e hipertensão pulmonar progressiva. Deve-se realizar correção das anomalias intracardíacas existentes ou, se há infecções recorrentes, ressecção do pulmão afetado. Devido à má evolução destes pacientes no pós-operatório, se não há sintomatologia ou "curto-circuito" sistêmico-pulmonar significativo o tratamento conservador é preconizado ${ }^{1}$.

Aqui, relatamos caso raro de pulmão em ferradura com acometimento de pulmão esquerdo, curso clínico tempestuoso e hipertensão pulmonar severa. Como não foi possível estudo anatomopatológico do caso em questão, alguns comentários se fazem necessários. Apesar de descrito de forma isolada como aqui apresentamos, o pulmão em ferradura é comumente relacionado à síndrome da cimitarra. Assim, de forma isolada, a origem aberrante da artéria pulmonar esquerda poderia caracterizar uma forma de anel vascular, como o sling da artéria pulmonar esquerda, mas sem compressão da traqueia distal. Poderíamos questionar também se o curso anormal desta artéria não seria uma variação da ramificação arterial pulmonar.

De qualquer maneira, consideramos importante a descrição do caso para que o reconhecimento desta doença seja possível em seu estágio precoce, pois apresenta associação com graus variáveis de hipertensão pulmonar de caráter evolutivo e, de forma geral, prognóstico precário.

\section{Potencial Conflito de Interesses}

Declaro não haver conflito de interesses pertinentes.

\section{Fontes de Financiamento}

O presente estudo não teve fontes de financiamento externas.

\section{Vinculação Acadêmica}

Não há vinculação deste estudo a programas de pósgraduação.

\section{Referências}

1. Frank JL, Poole CA, Rosas G. Horseshoe lung: clinical, pathologic, and radiologic features and a new plain film finding. AJR Am J Roentgenol. $1986 ; 146(2): 217-26$.

2. Lutterman J, Jedeikin R, Cleveland DC. Horseshoe lung with left lung hypoplasia and critical pulmonary venous stenosis. Ann Thorac Surg. 2004;77(3):1085-7.

3. Männer J, Jakob C, Steding G, Füzesi L. Horseshoe lung: report on a new variant - "inverted" horseshoe lung - with embryological reflections on the formal pathogenesis of horseshoe lungs. Ann Anat. 2001;183(3):261-5.

4. Freedom RM, Burrows PE, Moes CA. "Horseshoe lung: report of five new cases. AJR Am J Roentgenol. 1986;146(2):211-5.
5. Yoo SJ, Al-Otay A, Babyn P. The relationship between scimitar syndrome, so-called scimitar variant, meandering right pulmonary vein, horseshoe lung and pulmonary arterial sling. Cardiol Young. 2006;16(2):300-4.

6. Hawass ND, Badawi MG, Fatani JA, Meshari AA, Edrees YB. Horseshoe lung with multiple congenital anomalies: case report and review of the literature. Acta Radiol. 1987;28(6):751-4.

7. Matushita JPK, Missiaggia GC, Peixoto RMA, Leão Filho HM, Dias RS, Tavares Jr WC, et al. Pulmão em ferradura: relato de caso. Radiol Bras. 2007;40(5):359-61.

8. Ersoz A, Soncul H, Gokgoz L, Kalaycioğlu S, Tunaoğlu S, Kaptanoğlu M, et al. Horseshoe lung with left lung hypoplasia. Thorax. 1992;47(3):205-6. 